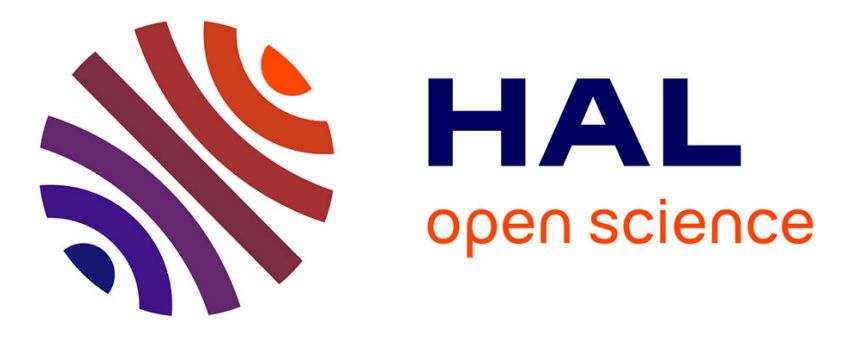

\title{
Collaborative agents for modeling traffic regulation systems
}

Neila Bhouri, Flavien Balbo, S Pinson, Mohamed Tlig

\section{To cite this version:}

Neila Bhouri, Flavien Balbo, S Pinson, Mohamed Tlig. Collaborative agents for modeling traffic regulation systems. The 2011 IEEE, WIC, ACM International Conferences on web intelligence and intelligent agent technology, Aug 2011, LYON, France. pp.7-13, 10.1109/WI-IAT.2011.62 . hal$00908897 \mathrm{v} 2$

\section{HAL Id: hal-00908897 \\ https://hal.science/hal-00908897v2}

Submitted on 21 Feb 2017

HAL is a multi-disciplinary open access archive for the deposit and dissemination of scientific research documents, whether they are published or not. The documents may come from teaching and research institutions in France or abroad, or from public or private research centers.
L'archive ouverte pluridisciplinaire HAL, est destinée au dépôt et à la diffusion de documents scientifiques de niveau recherche, publiés ou non, émanant des établissements d'enseignement et de recherche français ou étrangers, des laboratoires publics ou privés. 


\section{Collaborative agents for modeling traffic regulation systems}

\begin{tabular}{|c|c|}
\hline \multicolumn{2}{|c|}{ Neïla Bhouri ${ }^{1}$, Flavien Balbo ${ }^{1,2}$, Suzanne Pinson ${ }^{2}$ and Mohamed Tlig ${ }^{1}$} \\
\hline $\begin{array}{c}{ }^{1} \text { Université Paris Est, } \\
\text { IFSTTAR, GRETTIA, } \\
\text { "Le Descartes 2" } 2 \text { rue de la Butte Verte, } \\
\text { 93166 Noisy Le Grand Cedex. } \\
\text { neila.bhouri@ifsttar.fr }\end{array}$ & $\begin{array}{c}{ }^{2} \text { Université Paris-Dauphine } \\
\text { CNRS, LAMSADE, } \\
\text { Place du Marechal de Lattre de Tassigny F- } \\
75775 \text { Paris 16 Cedex, France. } \\
\text { \{balbo, pinson\}@lamsade.dauphine.fr }\end{array}$ \\
\hline
\end{tabular}

\begin{abstract}
The development of surface public transportation networks is a major issue in terms of ecology, economy and society. Their quality in term of punctuality and passengers services (regularity between buses) should be improved. To do so, cities often use regulation systems at junctions that grant priority to buses. However, most of them hardly take into account both public transport vehicles such as buses and private vehicle traffic. This paper proposes a bimodal urban traffic control strategy based on a multi-agent model. The objective is to improve global traffic, to reduce bus delays and to improve bus regularity in congested areas of the network. In our approach, traffic regulation is obtained thanks to communication, collaboration and negotiation between heterogeneous agents. We tested our strategy on a complex network of nine junctions. The results of the simulation are presented.
\end{abstract}

\section{Introduction}

To improve route times of public surface transportation (bus, tramways, shuttles, etc.), cities often use regulation systems at junctions that grant priority to vehicles. These systems are referred to systems equipped with bus priority. The aim of these strategies is to increase the average speed of public transport vehicles as well as other vehicles that has to cross a junction.

The use of these systems is efficient when traffic is light or when they need to improve a single congested bus route. However, reducing the time of bus journey, although very important for operating a route, is not the primary factor considered by public transport operators whose obligation is to provide passengers services e.g. keeping interval between buses. To take into account public transport vehicles specificity, TRSS
(Transportation Regulation Support Systems) were developed. TRSS systems follow a micro-regulation based approach, i.e. an approach that models the behavior of each bus [1], [2], [7]. One of the weaknesses of these systems is that the private vehicle traffic flow is hardly taken into account. Another weakness is that traffic light management which is one of the key factors of traffic jams and bus delays, is not included in the TRSS systems

Our objective is to build a traffic control strategy for bimodal traffic that is able to regulate both private vehicle traffic and public vehicle traffic. Classical control theory used to regulate bimodal traffic (public and private vehicles) is confronted to the modeling problem. Traffic flow can be modeled at a macroscopic or at a microscopic level. Microscopic modeling is timeconsuming, and it is therefore not well adapted to build real time control strategies for wide urban networks [14]. Macroscopic modeling has been used in [4], [5]. However, macroscopic representation of buses does not allow more than an indirect consideration of the intervals. In these systems, the objective was to reduce the time spent in traffic jams so that buses respect their schedule. In [11] a hybrid model was used macroscopic modeling for private vehicles and microscopic modeling for public transport. The complexity of the bimodal traffic regulation strategy shows the limits of these classical modeling approaches.

Multi-Agent modeling can be a suitable answer to this complex regulation problem. We note that multiagent systems are increasingly present in the field of traffic regulation [1] [2] [12] and [13]. The problem of traffic lights coordination on the thoroughfares of the route network has been solved in [8] [9] [10] and [15]. The regulation system presented in [16] is related to traffic assignment using negotiation between vehicles 
and junctions. We already developed a first prototype that shows promising results [6].

The second section focuses on traffic regulation systems and describes our model: the network model and the identification of the agents with a detailed description of agents, their attributes, their objectives, as well as communication and collaboration protocols. The third section provides the first results of the simulation tests carried out on the Jade platform. Finally, we conclude in the fourth section.

\section{Network modeling}

In our model, the urban network is represented by an oriented graph $\mathrm{G}=(\mathrm{I}, \mathrm{A})$. The nodes $\{\mathrm{I}\}$ represent the junctions (or intersections) and the $\operatorname{arcs}\{\mathrm{A}\}$ represent the lanes that connect the junctions. Two intersections can be connected by one or several arcs depending on the number of lanes on the thoroughfare.

An arc $a_{i}$ corresponds to a lane. It is characterized by a set of static information (such as its length $L_{i}$ (in meters), its capacity $C_{i}$ (maximum number of vehicles on $\operatorname{arc} \mathrm{a}_{\mathrm{i}}$ in private car unit, PCU), its saturation output $\mathrm{D}_{\mathrm{i}}$ which is the maximum exit output from the given arc (in PCU/second), and dynamic information i.e. the number of vehicles $\mathrm{N}_{\mathrm{i}}$ on the arc (in PCU), the state of the traffic lights at the extremity of the arc, green or red, if the light is green, then the vehicles present on the arc can depart. By private car unit (PCU), we mean that all vehicles on the arc are converted to their equivalent in private vehicles, for example a bus is 2.3 PCU depending on its length, a truck can be 2.3 or 4 PCU and so on.

A junction is specified by the set $E$ of arcs that enter it and $S$ the set of arcs that leave from it. A junction is managed by a set of stages $P$. Each of the stages specifies the list of arcs for which the green light is awarded if the stage is active (see Figure 1). The network is used by a number of bus routes.

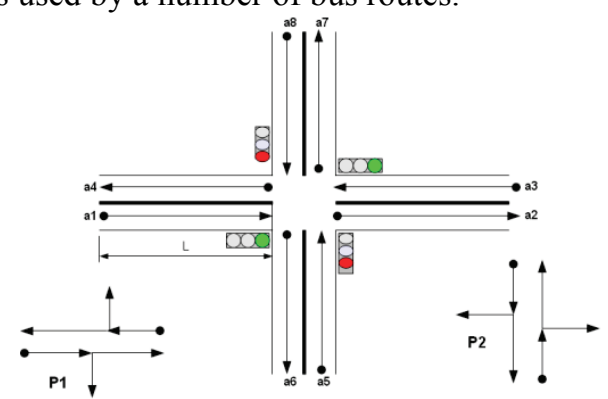

Figure 1: Example of a junction with 4 arcs and two stages $P=\{P 1, P 2\}$. $P 1$ allows for the clearing of the arcs a1 and a3, because the entry flow a1 and a3 can leave the junction at the same green light period. Simi- larly, P2 clears arcs a5 and a8. The entries and exits of the junction are respectively $E=\{a 1, a 3, a 5, a 8\}$ and $\mathrm{S}=\{\mathrm{a} 2, \mathrm{a} 4, \mathrm{a} 7, \mathrm{a} 6\}$.

Each route comprises the number of buses from the same origin and in the same direction, and that service a number of predefined commercial bus stops at regular time intervals. The time spent by a bus at a commercial stop is equal to the pre-set time for passengers to mount, plus additional time to regulate the interval, if required.

\subsection{Agent modeling}

In order to identify agents and design the MAS, we present an abstraction of the real system; for every entity of the real world is associated an agent in the virtual world to form a Multi-Agent System (MAS). Homogenous agents are called "agent-type". The developed MAS is made up of the following agenttypes:

Junction Agent (JA): is the key agent of our architecture. It is in charge of controlling a junction with traffic lights, and of developing a traffic signal plan. The junction agent modifies the planning of the lights according to data sent by approaching buses.

Stage Agent (SA): the traffic signal plan is elaborated thanks to the collaboration of the junction and stage agents. Each SA is expected to determine the optimal green light split to clear the waiting vehicles on the arcs concerned by the stage. Thus, whatever the complexity of the junction is (and its physical configuration), it is managed by a set of stage agents interacting with the junction agent in order to develop a plan of actions for the traffic lights. The number of stage agents is given a priori depending of the junction topology.

Bus Agent (BA): represents a bus in the real world. It circulates from one arc to another, halts at commercial stops, halts at red lights and obeys the instructions of the bus route agent. The objective of each bus agent is to minimize the time spent at traffic lights (to minimize journey times).

Bus Route Agent (BRA): bus agent only provides a local view of their environment and, in particular, only the journey covered by the BA. Thus, local optimization carried out by bus agents can have a negative impact on the route, notably on its regularity (i.e. the formation of bus queues). To tackle this problem, we propose an agent who has a global view of the route agents, and who can control and modify their behavior in order to guarantee an efficient and regular service. 


\subsection{Description of agent behavior}

Bus Agent (BA): In order to minimize the time spent at traffic lights the bus agent interacts with junction agents and its hierarchical superior agent (BRA). All the buses have to provide a regular service and avoid bus queues, in other words, the frequency of buses passing commercial stops must remain stable. To achieve this objective, the BA receives orders from the BRA (for example, stay at the stop for t seconds, if the bus is ahead with respect to the position of the preceding bus).

The BA is composed of a data module, which represents its internal state, and a communication module, that enables exchange with other system agents.

Behavior of a bus agent: Let $\mathrm{t}_{0}$ be the entering time of the bus agent which behaves in the following way:

- On entering arc $\mathrm{i}$, the BA retrieves information from the arc (the number of vehicles that precede it, the length, capacity, and exit output of the arc). By using these data, the BA calculates a time-space request that is transmitted to the JA in order to prevent an eventual stop at the red light at the following junction. The JA then attempts to satisfy the demand (see junction agent below);

- When approaching a stop, the BA informs the associated BRA. The bus route agent then calculates the duration of the regulation interval and its level of priority and sends it to the bus. Priority level is a function of the predecessor bus delay: the greater the delay, the higher the priority. The bus must wait during the passenger loading time, as well as the potential regulation time, before leaving the stop.

Calculation of a green light request. This calculation is specified by the interval of time during which the green light is granted to the actual arc so that the bus can pass without stopping at the next junction. Let $R$ be the requested interval: $R=\left[t_{b}, t_{e}\right]$, with $t_{b}$ and $t_{e}$ the beginning and ending times of the request interval respectively. The calculation of these times is carried out as follows: the bus enters the arc and finds $N_{V}$ vehicles ahead of it, the vehicles move to the traffic lights lane to wait for the green light thus forming a queue of length $F$ (see Figure 2). In order to continue along its route, the queue of vehicles has to be dispersed before it arrives. The green light should thus be granted at the arc at the instant: $t_{b}=t_{o}+T-T_{F}$ with $T$ be the time necessary for the bus to cover the distance Dis between the beginning of the arc and the end of the queue, and $T_{F}$ be the time necessary to disperse the queue $F$.
This request interval $R$ together with other information (id-number of the bus, its priority, the actual arc of the bus, the next arc to be traveled by the bus) are sent to the JA (at the next junction) who attempts to modify the plan for the lights to satisfy the request.

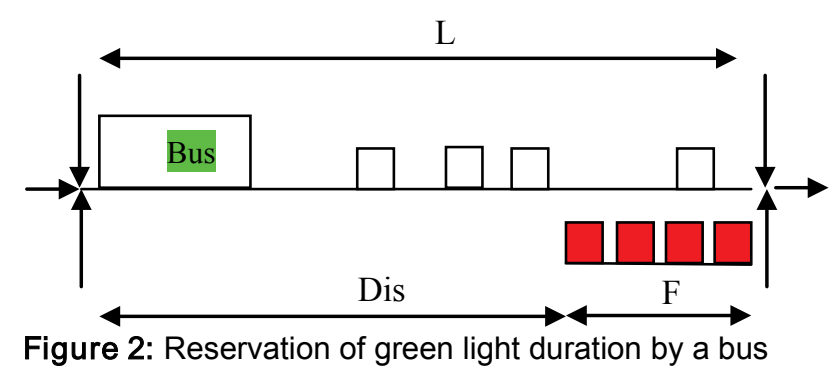

Junction Agent (JA): The JA is the key agent of our architecture. The JA supervises the group of stage agents (SA) who collaborate together to establish a plan for traffic lights. This plan will, on one hand, maximize the capacity of the junction and, on the other hand, attempt to satisfy, as far as possible, the request interval of the bus. The JA is characterized by static and dynamic data.

The static data represent the constraints that characterized the JA. It contains the maximum value of the traffic light cycle (120 seconds). For each cycle, there is an interval of lost time i.e. the period of orange or all red. The all red light is a period during which all the arcs from the same junction have a red light in order to clear the centre of the junction and thus prevent accidents. This fixed period, in conformity with the architecture of the junction, does not depend on the length of the cycle. It is fixed here to a two second period after each stage. It contains also the set of stages of the junction: $P=\left\{P_{l}, \ldots, P_{m}\right\}$. The set of stages represents the configuration of the junction (the permitted movements and turns). Determining the stages is a task executed offline by the traffic experts.

They are two types of dynamic data, the first is related to the traffic signal plan: it specifies the order of the stages as well as duration of each stage. Between two successive stages, a two second period of all red is imposed. The second is related to the list of received reservation request data from the bus agents: each request is specified as follows: $R=\left(P_{i}, t_{b}, t_{e}\right.$, Priority $)$, where $P_{i}$ is the stage that will allow the passage of the given bus, $t_{b}$ the time when the bus is expected to arrive at the traffic light, $t_{e}$, the time when the rear of the bus leaves the arc, and finally Priority is the level of bus priority defined by the bus route agent. 
At the end of each cycle, the JA triggers the process of calculating the traffic signal plan for the given cycle. This signal plan determines the duration of the green light and the ranking of each stage. When the JA receives a request from a bus, it records it in the database. The JA then decides to accept or to refuse this request at time $t_{b}$. The modification of a traffic signal plan following a priority request by a bus is as follows: 1) Extension or reduction of a stage (delay or advance), without exceeding the maximal duration of a stage; 2) Introducing a new stage into the plan. It is explained thanks to the following example (see Figure 3) in which the junction has a plan with four stages and two antagonistic bus routes.

In this example, in the initial traffic signal plan, the order of stages is $P_{2}$ during 20 seconds, $P_{1}$ and $P_{3}$ during 30 seconds each and finally $P_{4}$ for 20 seconds. At time $t_{1}$, the JA receives a first request $R_{1}\left(P_{2}, t_{3}, t_{4}, 2\right)$ which means that bus $\mathrm{n}^{\circ} 1$ is asking for stage $P_{2}$. It needs a green light at this stage during the interval $\left[t_{3}, t_{4}\right]$ and it has a priority index of " 2 ". The JA doesn't plan this request immediately but it waits until the start time $t_{3} . R_{1}$ is studied at time $t_{3}$ (start time) and not at time $t_{1}$ (received time), because during this delay, the JA may receive more reservations. In this example, at instant $t_{2}$ the junction agent receives another reservation, $R_{2}$ from bus $n^{\circ} 2$ which has a level of priority equal to 4 and requests the stage $P_{4}$. As it is not possible to satisfy both requests because they involve two different stages $\left(P_{4}\right.$ and $\left.P_{2}\right)$ for two time intervals that overlap, JA gives the stage to the bus with the highest priority index. In our example, $R_{1}$ is refused because reservation $R_{2}$ is more prioritized. This planning process is fundamental to regulate bus intervals.

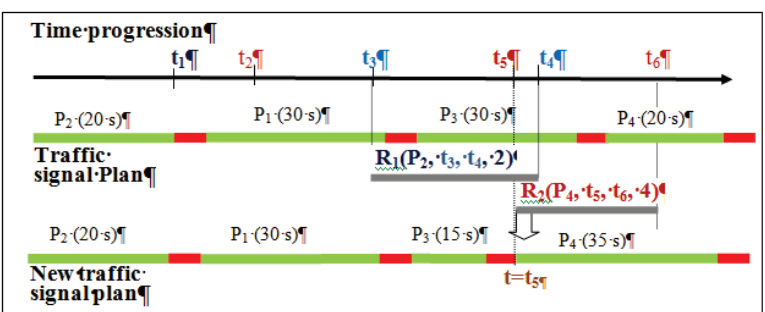

Figure 3: Example of a traffic signal plan and its modification

Calculation of a traffic signal plan. The plan is calculated through the collaboration of the junction agent (JA) and the Stage Agents (SAs). The JA plays the role of a manager in supervising the SAs that act as participants. The protocol follows by the junction agent $\mathrm{JA}$ is as follows:
1- JA interacts with its stage agents (SA) that needs to be managed. Let's call this set of SAs collab-group.

2- Initialization of variables:

$C=C y c l e M a x, C$ is the size of the calculated cycle (in the example $C=120$ s).

$t=0$

3- JA sends a message inform to the stage agents to inform them of the protocol initiated to calculate the traffic signal plan.

4- JA sends a message request to the stage agents asking them for the time necessary to clear all the vehicles from their stages, beginning at instant $t$.

5 - Each stage agent $i$ calculates its desired green light duration $d_{i}$ and an index that measures the urgency $I_{i}$ of the stage, and sends them to the manager (see next page for $I_{i}$ and $d_{i}$ calculation).

6- When JA receives all the responses, it calculates the sum $d$ of durations.

If $d>C$ then the JA has to solve a conflict since the size of the cycle exceeds the maximum. Conflict is solved when d previously calculated becomes less or equal to $C$ : $d \leq C$ (see conflict resolution protocol on Figure 4).

7- JA selects $P_{j}$, the most urgent stage; let $d_{j}$ be its duration.

8- JA sends an accept message to the stage agent in charge of operating this stage.

9- JA withdraws the corresponding stage agent from collab_group.

10- JA updates the variables $C=C-d ; t=t+d_{j}$;

11- If collab_group is not empty, the protocol returns to step 4 .

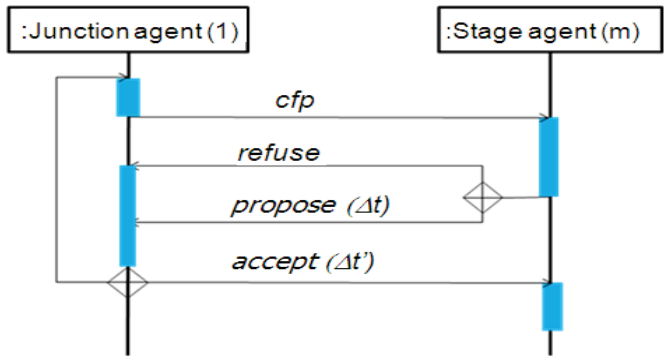

Figure 4: Collaboration protocol in conflict resolution

Conflict resolution. When the sum of green light durations requested by stage agents exceeds the size of the accepted value of the cycle, the JA must restore this sum to the maximal value of the cycle. To achieve a $\Delta t$ reduction, at minimum cost, the manager negotiates with the JA using a Contract Net Protocol. The 
cost of the offer is the number of buses penalized if the stage agent reduces its duration of $\Delta t$ (see Figure 4).

The conflict resolution protocol is as follows:

1- JA initializes cost c: $c=1$;

2- JA sends a call_fo_propose (cfp) message with a proposition and cost $c$ to all the stage agents associated with the junction

3- The stage agents reply with a propose or refuse message (modalities are explained in the state agent section below)

4- Once all the responses have been received by the JA, all the offers are added.

5- If this sum is less than the cycle size, then the conflict is solved. Otherwise, JA increments the cost c, sends a new proposition d' and returns to step 2 .

Stage Agent (SA): This agent has a collection of both static and dynamic data that represents its internal state. The Static data are the list of entry arcs, the list of arcs authorized to clear if the stage is active (or green)

Dynamic data are related to 1) the state of the stage: active or inactive; 2) the duration of green light attributed to the stage; 3 ) the starting time of stage execution. 'Active' means that the traffic lights controlling the arcs concerned by this stage are green. The vehicles are therefore authorized to depart.

Behavior of the stage agent. The SA participates in the calculation of the traffic signal plan, and is in charge of fixing the optimal duration of green light for the given stage. When the stage agent is asked about the desired duration of green light by the junction agent, this duration $d_{i}$ and an index $I_{i}$ that measures the urgency of the stage, are computed and transmitted to the junction agent. If the stage agent receives confirmation from the junction agent, the stage agent stops the process. If the stage agent receives a cfp (call for propose) with a cost $c$, it computes an offer and sends it to the junction agent. light

\section{Calculation of the desired duration of the green}

The optimal duration of green light is computed by the following formula:

$$
T=\max _{i=1, \ldots, m}\left\{T_{i}\right\} \quad T_{i}=w_{i} \frac{\mathrm{N}_{\mathrm{i}}}{\mathrm{D}_{\mathrm{i}}}+\left(1-w_{i}\right) \cdot \frac{\mathrm{N}_{\mathrm{i}} * \mathrm{~L}_{\mathrm{i}}}{\mathrm{C}_{\mathrm{i}} * \mathrm{~V}_{\mathrm{i}}}
$$

where $m$ is the number of entering arcs at this stage, $T_{i}$ the time necessary to clear arc $i, L_{i}$ the length (meter), $V_{i}$ the average speed (meter/second), $N_{i}$ the number of vehicles, $D_{i}$ is the saturation flow and $C_{i}$ the capacity of the arc $i$. The number of vehicles $N_{i}$ and the capacity $\mathrm{Ci}$ are expressed in private car unit (PCU)

$W_{i}=N_{i} / C_{i} \in[0,1]$ is a parameter that indicates the degree of congestion of the arc. When the arc is congested, $W_{1}=1$, which means that only the first part of the equation is used.

Urgency index of a stage. To award priority to a bus, the urgency index of a stage $j$ is defined by the fact that the higher the index, the greater the urgency of the stage:

$$
I_{j}=\sum_{i=0}^{m}\left(e^{w_{i}}+e^{b_{i}}\right)
$$

with: $w_{\mathrm{i}}$ the parameter indicating the degree of congestion of arc $I, b_{i}$ is the number of buses present on arc $i, m$ is the number of arcs entering via stage $j$ and $e$ is the Euler constant in our example. We can note that if there are several buses on arc $\mathrm{i}$ (if $b_{i}>1$ ), the term $e^{b i}$ is dominant and therefore gives priority to stages with buses; if $b_{i}=0$, the degree of congestion is then taken into account.

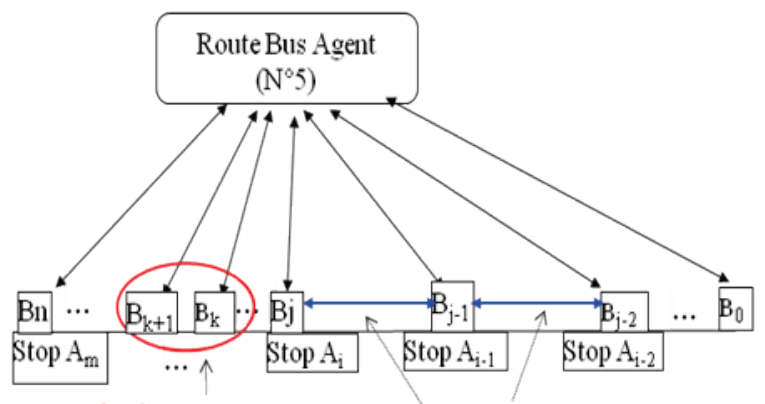

\section{$\mathrm{Pb}$ : bus queue Regular intervals}

Figure 5: Supervision of bus agents (BA) by the route agent (BRA).

Bus Route Agent (BRA). The role of the route agent is to supervise bus agents so as to prevent a local level regulation and the creation of bus queues. In other words, this agent can modify the behavior of bus agents in two different ways: 1) directly. by keeping hose buses, which are ahead in the plan compared to the preceding ones, at the bus stop for a certain period of time; 2) indirectly. by modifying bus priorities. This agent has a global view of the route it operates on, and can therefore detect bus queues and react to prevent queue formation. 
Internal state of the route agent. The route agent encompasses the following data: 1) the set of arcs traveled by the bus on its route; 2) The set of stops on the route: for each stop, its position, and the distance separating it from the next stop; 3 ) The set of buses on the route; 4) The frequency of buses introduced onto the route. For two consecutive stops $A_{i-1}$ and $A_{i}$, the route agent maintains the journey time $\mathrm{tt}_{\mathrm{j}}$ of the last bus. This helps to follow the bus journey and to calculate whether the bus is ahead or late compared to the bus immediately preceding it.

Behavior of the route agent. When a bus $\mathrm{j}$ moves to a stop, the time $t_{j}$ taken to cover the distance that separates the two stops $A_{i}$ and $A_{i-1}$, is transmitted to the route agent. The route agent then compares $t t_{j}$ to the time $\left(t t_{j-1}\right)$ taken by its preceding bus and consequently decides whether the bus is ahead or late. The route agent computes the new priority of the bus agent as well as the length of time the bus should wait at the commercial stop if it is ahead [6].

\section{Experimentation and results}

To test our bimodal control strategy, we developed a Multi-Agent System prototype on the $\mathrm{JADE}^{1}$ platform (Java Agent Development Framework). JADE offers Java middleware based on a peer-to-peer architecture with the overall aim to provide a runtime support for agents

We have tested the strategy on a network of nine intersections (Figure 6):

- The distance between two adjacent junctions belongs to $[200,400]$ meters.

- Each section comprises one or two lanes.

- The saturation flow, which is the maximum exit output of the arcs, is the same for each arc and equal to 0.5 vehicle/second.

- At each entry onto the network, we have installed a source that generates vehicles at a frequency $F \in[4 \mathrm{~s}$ ... $10 \mathrm{~s}]$.

- Some of the junctions have two stages while others have three stages.

- Two buses enter the network. For Bus Route 1, the frequency of the generated buses is 50 seconds and for Bus Route 2, the frequency is 40 seconds.

Knowing that bus priority can have negative impact on the global traffic conditions and can hence have negative impact on buses traffic, we tested our MAS strategy in two cases. In the first case, we use the

1 jade.tilab.com/
MAS strategy as presented above. In the second case, we inhibit the bus priority function. This last case will be called the "without priority" strategy.

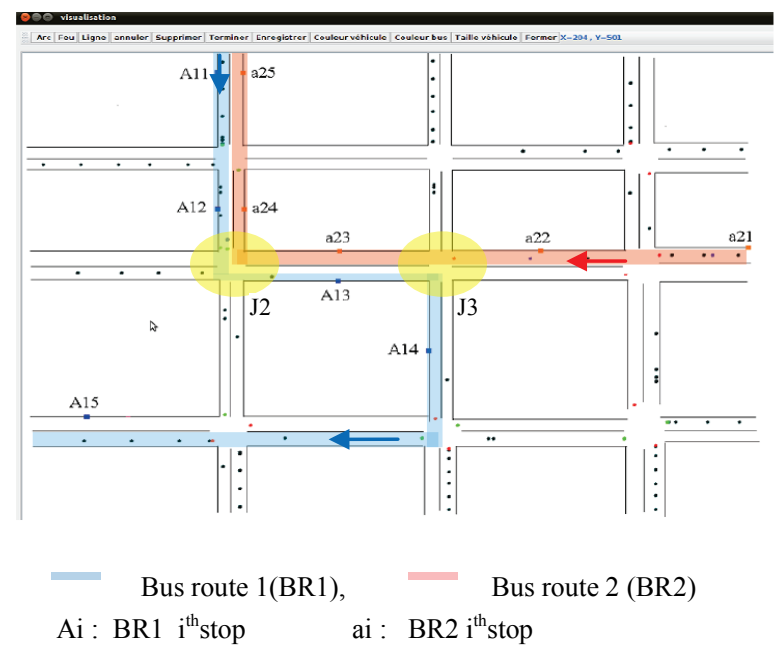

Figure 6: The simulated network

\subsection{Simulation results at network level}

Figures 7 gives simulation results of the three strategies for very heavy traffic conditions. Figure 7.a shows the recorded delays for buses with the three control strategies and Figure 7.b shows the same kinds of curves for private vehicles.

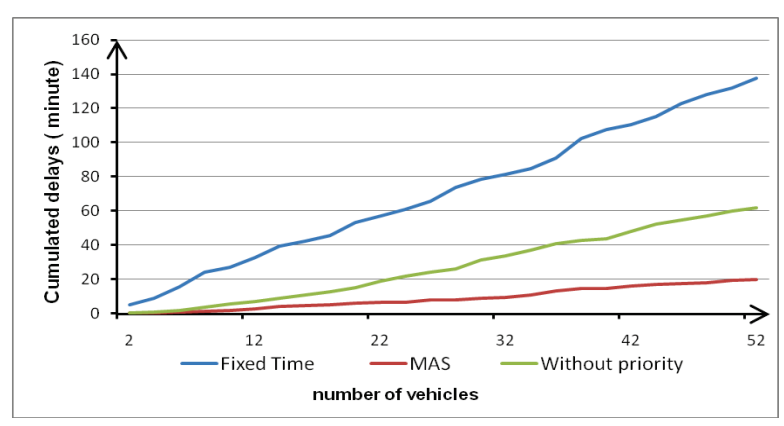

Figure 7.a: Buses cumulated delays

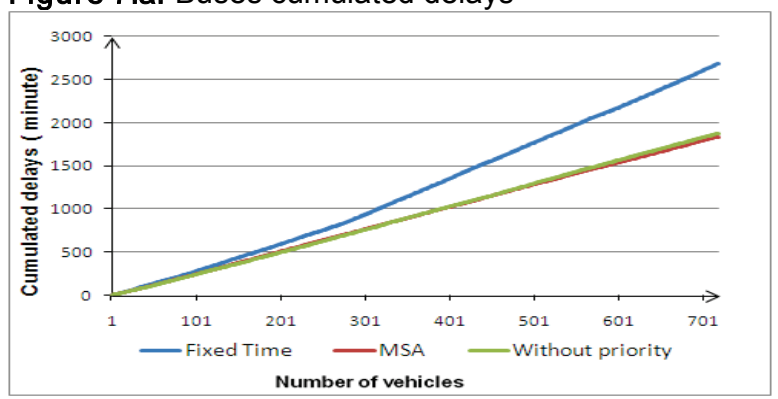

Figure 7.b. Private vehicles cumulated delays 
These delays correspond to the sum of time lost by all buses (resp. vehicles) during stops at traffic lights. Figure 7.a shows that, on the simulation period, the MAS strategy improves bus travel time (cumulated bus delays) of $85 \%$ compared to the Fixed Time Strategy (FTS) whereas the "Without priority" strategy improves buses traffic of only $76 \%$. Furthermore, this last FTS strategy doesn't reduce private vehicles delays (see Figure 7.b). Figure 7.b shows interesting results: "without priority" strategy and MAS strategy give same cumulated delays for private vehicles. They both improve vehicles delays by $30 \%$. Thanks to the MAS strategy, the average lost time by bus is equal to 23 seconds, when it is equal to 2.6 minutes with the Fixed Time strategy. Considering these two results, we can conclude that MAS strategy is the best one since it improves significantly buses traffic as well as private vehicles traffic, and, using bus priority, helps traffic regulation.

\subsection{Simulation results at junction level}

In this section, we study the MAS strategy at a microscopic level: the junction level. We choose $\mathrm{J} 2$ and $\mathrm{J} 3$ junctions (see Figure 6). Each of them has 3 phases. In J2, bus lines use arcs with different phases. This means that the two bus lines are conflicting; if two buses at the same time ask for priority, junction J2 will have to solve a conflicting situation as explained in Figure 3. In J3, bus lines are not conflicting although junction $\mathrm{J} 3$ has also 3 phases. Buses run during the same phases.

\begin{tabular}{|c|c|c|c|c|c|c|c|c|}
\hline \multirow{2}{*}{ Strategies } & \multicolumn{4}{|c|}{ BR1 } & \multicolumn{4}{c|}{ BR2 } \\
\cline { 2 - 9 } & \multicolumn{2}{|c|}{ J2 } & \multicolumn{2}{c|}{ J3 } & \multicolumn{2}{c|}{ J2 } & \multicolumn{2}{c|}{ J3 } \\
\hline MAS & 29.7 & $27 \%$ & 28.7 & $14 \%$ & 29.4 & $28 \%$ & 27.2 & $5 \%$ \\
\hline $\begin{array}{c}\text { Without } \\
\text { priority }\end{array}$ & 36.6 & $10 \%$ & 28.7 & $14 \%$ & 31.5 & $22 \%$ & 27.8 & $3 \%$ \\
\hline $\begin{array}{c}\text { Fixed } \\
\text { Time }\end{array}$ & 40.6 & - & 33.2 & - & 40.6 & - & 28.7 & - \\
\hline
\end{tabular}

Table 1: Average travel time (TT) between the two bus stops around the junctions J2 and J3 for BR1 and BR2. TT is expressed in seconds.

Table 1 gives the average travel time for vehicles on BR1, first between bus stops A12 and A13, showing the impacts of the three strategies on junction $\mathrm{J} 2$ traffic, and then between stops A13 and A14, showing the situation on junction J3. For vehicles on BR2, travel time is measured between a 23 and a 24 and then between a22 and a23. One can notice that the MAS strategy improves bus travel time even for junction J3, where as explained before, buses can cross $\mathrm{J} 3$ on two stages among the three ones available and hence can cross quickly. We can also notice that MAS strategy succeed to suppress the delays on this junction, as if there is no congestion, the minimum time needed to travel the 300 meters separating the two bus stops a22 and a23 is 27 seconds. On junction J2, where buses can run only on one stage, benefits on travel time are more important. They are equal to $27 \%$ for BR1 and $28 \%$ for BR2.

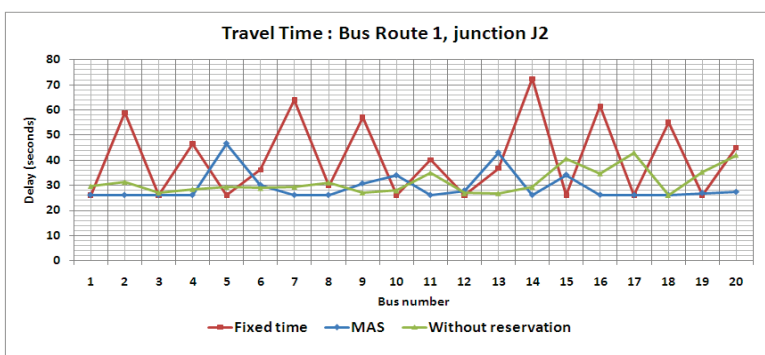

Figure 8.a: Buses travel time on BR1 from A12 to A13

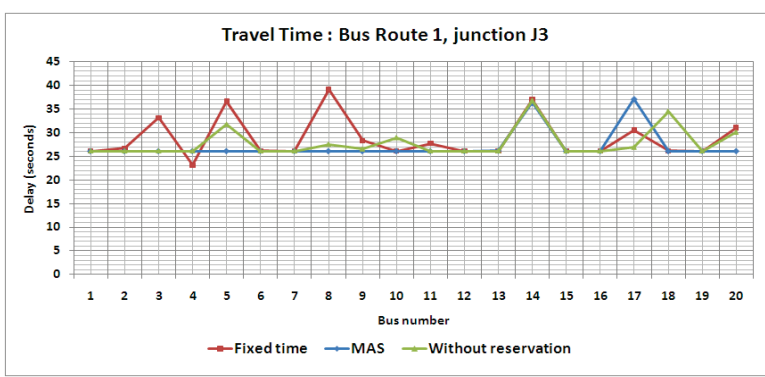

Figure 8.b: buses travel time on BR1 from $A 13$ to $A 14$

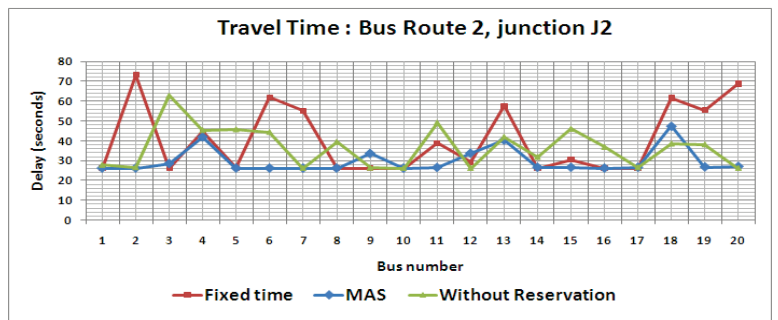

Figure 9.a: buses travel time on BR2 from a23 to a24

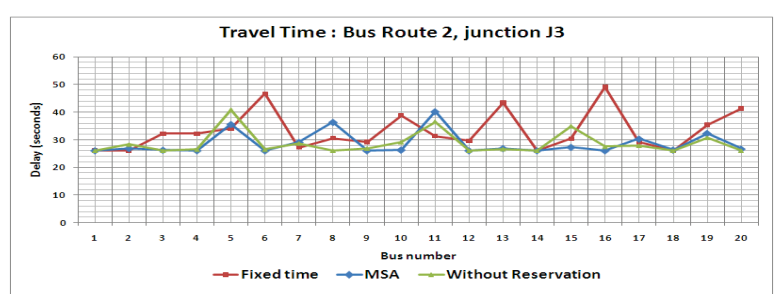

Figure 9.b: Buses travel time on BR2 from a22 to a23 
Figure 8 (respectively Figure 9) gives bus delays for BR1 (respectively BR2) buses at junction J2 and J3. The $\mathrm{X}$ axis represents the bus numbers generated by the simulation. We can see on these figures that another advantage of MAS strategy is that it preserves regularity of travel time (delays stay nearly constant) across these junctions.

\section{Conclusion}

In this paper, we have developed a bimodal traffic control strategy based on a multi-agent system. Unlike other approaches, our model takes into account both public transport vehicles such as buses and private vehicle traffic and studies the regulation in a whole network. The objective of this research was to improve global traffic, to reduce bus delays and to improve bus regularity in congested areas (keeping regular interval between buses) of the network. In our approach, traffic regulation is obtained thanks to communication, collaboration and negotiation between heterogeneous agents at different levels of abstraction and at different level of granularity (microscopic vs macroscopic level). Firstly, we have shown that classical methods of traffic regulation present several weaknesses. Secondly, we have presented our multi-agent strategy that computes traffic signals plans based on the actual traffic situation and on priority given to buses. Thirdly, we have run a simulation prototype on the JADE platform. The results show that our MAS strategy with priority improves both buses travel time and buses regularity. Our results also show that this bimodal MAS strategy improves buses as well as private vehicles traffic and reduces bus delays. Further work needs to be done: a more realistic network should be defined in the simulation run and more validation and testing should be undertaken with the definition of several indicators. It would also be interesting to have more testing to find Pareto front and multi-criteria optimization in order to get equilibrium between public transport delays and private traffic delays

\section{References}

[1]. Balbo, F., Pinson, S., 2010. "Using intelligent agents for Transportation Regulation Support System design", Transportation Research Part C: Emerging Technologies, Volume 18 (1), pp 140-156.

[2]. Balbo, F., Pinson, S., 2005. "Dynamic modeling of a disturbance in a multi-agent system for traffic regulation", Decision Support Systems 41(1), pp 131-146

[3]. Ana L. Bazzan. 2009. Opportunities for multiagent systems and multiagent reinforcement learning in traffic control., Autonomous Agents and Multi-Agent Systems 18 (3), pp 342-375.

[4]. Bhouri, N., 2009. Constrained Optimal Control strategy for multimodal urban traffic network. IFAC Workshop on Control Applications of Optimization (CAO'09), Finland.

[5]. Bhouri, N., Lotito P., 2005. "An intermodal traffic control strategy for private vehicle and public transport". 10th Euro Working Group on Transportation, Poznan, Poland.

[6]. Bhouri, N., Haciane S., Balbo F., 2010. "A MultiAgent System to Regulate Urban Traffic: Private Vehicles and Public Transport”. 13th IEEE-ITSC, Portugal, pp. 15751581.

[7]. Cazenave T., Balbo F., Pinson S., 2009. "MonteCarlo Bus Regulation", $12^{\text {th }}$ Int. IEEE ITSC'09, St. Louis, MO, USA, pp. 340-345.

[8]. De Oliveira D, Bazzan A.L., Lesser V, 2005. "Using Cooperative Mediation to Coordinate Traffic Lights: a case study", AAMAS'05, New York, NY, USA, pp 463-470.

[9]. Ferreira, E.D., Subrahmanian, E., Manstetten,D., 2001. "Intelligent agents in decentralized traffic control", IEEE Intelligent Transp. Systems Conf. IEEE-ITSC'01Oakland (CA), USA - August 25-29, pp 705-709.

[10].France, J., Ghorbani, A., 2003. "A multiagent system for optimizing urban traffic". In Proceedings of the IEEE/WIC Inter. Conf. on IAT. Washington, DC: IEEE Computer Society, pp. 411-414

[11].Kachroudi, S. and Bhouri N., 2009. “A multimodal traffic responsive strategy using particle swarm optimization", 12th IFAC Symposium, Redondo Beach, California, USA.

[12].Mailler R. and Lesser V, 2004. "Solving distributed constraint optimization problems using cooperative mediation”, AAMAS'04, IEEE Computer Society, pp 438-445

[13].Mizuno, K., Y. Fukui and S. Nishihara, 2008. "Urban Traffic Signal Control Based on Distributed Constraint Satisfaction", Proceedings of the 41st International Conference on System Sciences. Hawaii, pp 65.

[14].Papageorgiou, M., Ben Akiva, M., Bottom, J., Bovy, P., Hogendoorn, S., Hounsell, N., Kotsialos, A. \& McDonald, M. 2007. ITS and Traffic Management. In Handbook in OR\&MS: Transportation. Barnhart, C. \& Laporte, G. North-Holland. 715 - 774.

[15].Roozemond D. A., 2001. "Using intelligent agents for pro-active, real-time urban intersection control", European Journal for Operational Research 131, pp 293-301.

[16].Vasirani, M., Ossowski, S., 2009. "A marketinspired approach to reservation-based urban road traffic management”. AAMAS'09, volume 1, pp 617-624. 